University of Wollongong

Research Online

Faculty of Engineering - Papers (Archive)

Faculty of Engineering and Information

Sciences

$1-1-2007$

Impurity mediated in absorption continuum in single walled carbon nanotubes

Chao Zhang

University of Wollongong, czhang@uow.edu.au

J. C. Cao

Chinese Academy of Sciences

Xiao-Bing Guo

Chinese Academy of Sciences, guoxba@lenova.com

Feng T. Liu

University of Utah, fliu@uow.edu.au

Follow this and additional works at: https://ro.uow.edu.au/engpapers

Digitart of the Engineering Commons

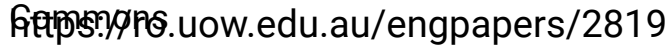

Network

Logo

Recommended Citation

Zhang, Chao; Cao, J. C.; Guo, Xiao-Bing; and Liu, Feng T.: Impurity mediated in absorption continuum in single walled carbon nanotubes 2007, 023106-1-023106-3.

https://ro.uow.edu.au/engpapers/2819

Research Online is the open access institutional repository for the University of Wollongong. For further information contact the UOW Library: research-pubs@uow.edu.au 


\title{
Impurity mediated absorption continuum in single-walled carbon nanotubes
}

\author{
C. Zhang a) \\ School of Engineering Physics, University of Wollongong, New South Wales 2522, Australia \\ J. C. Cao and X. G. Guo \\ State Key Laboratory of Functional Materials for Informatics, Shanghai Institute of Microsystem \\ and Information Technology, Chinese Academy of Sciences, Shanghai 200050, China \\ Feng Liu \\ Department of Materials Science and Engineering, University of Utah, Salt Lake City, Utah 84112
}

(Received 6 November 2006; accepted 6 December 2006; published online 9 January 2007)

\begin{abstract}
The authors demonstrate that in single-walled carbon nanotubes, a weak impurity potential can lead to a strong above-gap absorption continuum. The total absorption is enhanced due to the intraband and indirect transitions, as well as plasmon excitations, which are forbidden in perfect nanotubes. Such impurity induced absorption is strongly dependent on the size and chirality of the tube. (C) 2007 American Institute of Physics. [DOI: 10.1063/1.2431444]
\end{abstract}

Single-walled carbon nanotubes (SWNTs) possess many interesting electronic properties, making them attractive for potential applications in electronic and optoelectronic devices. Their electronic properties display a strong dependence on their size and chirality ${ }^{1}$ as well as a high sensitivity on defects, ${ }^{2}$ impurities, ${ }^{3}$ and structural deformations. ${ }^{4,5}$ They have been used as channels in model transistors ${ }^{6,7}$ and have been proposed for building electromechanical sensors. ${ }^{8}$ Furthermore, the photon absorption spectra of SWNTs have been exploited to identify their types. ${ }^{9-11}$ There have been considerable interest in using SWNTs to make photodetectors and photovoltaic devices. ${ }^{12-17}$

The optical properties of SWNTs are expected to be highly sensitive to defects and impurities, ${ }^{13,14}$ which will play an important role in their use of optoelectronic devices. So far, however, theoretical calculations, especially those based on first-principles method, ${ }^{18-21}$ have been limited to perfect SWNTs. However, the real SWNT samples are generally not perfect, but experience various electronic perturbations due to structural deformation, topological defect, impurity, intertube interaction in SWNT bundles and films, and electron-phonon interaction. These perturbations can induce intraband and indirect transitions, as well as plasmon excitations. In this letter we analyze the effect of impurity scattering on the optical absorption in SWNT. Our calculated photoresponses of SWNTs show a large absorption continuum, which increases with increasing strength of impurity scattering. We demonstrate that the continuous photon absorption has the impurity mediated plasmon excitations as its origin.

To calculate photon absorption of SWNTs, we employ a quantum transport equation in the presence of both electronelectron (e-e) and electron-impurity (e-i) scattering. We treat the various e-i scattering mechanisms in the most general manner by a random potential within the tight-binding (TB) formalism. Previous studies at finite frequencies are limited to the electronic polarizability, ${ }^{22-25}$ and existing theories on electron transport in SWNT are mostly limited to the case of zero frequency. Our approach takes into account the effects

\footnotetext{
${ }^{a)}$ Electronic mail: czhang@uow.edu.au
}

of plasmons and other electronic excitations on the optical absorption. The excitons can affect the absorption characteristics near and below the energy gap, but have a negligible effect on the absorption continuum at high frequencies. Therefore we shall neglect the exciton effect in this work.

The Hamiltonian of the system can be written as $H$ $=H_{0}+H_{\mathrm{ee}}+H_{\mathrm{ei}}+H_{\mathrm{e} \gamma}$, where $H_{0}$ is the Hamiltonian of noninteracting electrons in a SWNT, $H_{\mathrm{ee}}$ is the e-e interaction, $H_{\mathrm{ei}}$ is the e-i interaction, given as $\sum_{i} U\left(\mathbf{r}-\mathbf{R}_{i}\right)$, where $\mathbf{R}_{\mathrm{i}}$ is the position of the $i$ th impurity, and $H_{e \gamma}$ is the coupling of electrons to the electrical field $\mathbf{E}(t)=\mathbf{E} \exp (-i \omega t)$. The single electron wave functions are given as $\psi^{c, v}\left(k_{x}, k_{y}\right)=(1 / \sqrt{2})$ $\times\left[\phi_{1}(\mathbf{k}, \mathbf{r}) \pm\left[H_{12}^{*}\left(k_{x}, k_{y}\right) /\left|H_{12}\left(k_{x}, k_{y}\right)\right|\right] \phi_{2}(\mathbf{k}, \mathbf{r})\right]$, where $\phi_{1}$ and $\phi_{2}$ are the TB basis functions, and the nearest-neighbor Hamiltonian matrix element, $H_{12}=\gamma_{0}\left\{e^{i k_{x} a / \sqrt{3}}\right.$ $\left.+2 e^{-i k_{x} a / 2 \sqrt{3}} \cos \left(k_{y} a / 2\right)\right\}$, where $a=2.46 \AA$ and $\gamma_{0}=3.03 \mathrm{eV}$. The energy dispersion of an $(m, n)$ SWNT (Ref. 1) is $\epsilon\left(k_{x}, k_{y}\right)$. The $x$ component of $\mathbf{p}$ is quantized $p_{x}=J / r$, with $r=a \sqrt{\left(m^{2}+n m+n^{2}\right)} / 6 \pi$ for an $(m, n)$ tube.

We define $F_{n n^{\prime}}(\mathbf{k}, \mathbf{k}+\mathbf{q})=\left\langle a_{\mathbf{k}, n}^{\dagger}(t) a_{\mathbf{k}+\mathbf{q}, n^{\prime}}(t)\right\rangle$ as the density matrix between the state $\langle n, \mathbf{k}|$ and $\left|n^{\prime}, \mathbf{k}+\mathbf{q}\right\rangle$. Here $a_{\mathbf{k n}}^{\dagger}\left(a_{\mathbf{k n}}\right)$ is the creation (annihilation) operator for an electron in the state $|n, \mathbf{k}\rangle$. The equation of motion for the density matrix is given as

$$
\begin{aligned}
\left(i \partial F_{n n^{\prime}} / \partial t\right)(\mathbf{p}, \mathbf{p}+\mathbf{k})= & {\left[\boldsymbol{\epsilon}_{n}\left(J, p_{y}\right)-\epsilon_{n^{\prime}}\left(J+L, p_{y}+k_{y}\right)\right.} \\
& \left.+(i e \mathbf{k} \cdot \mathbf{E} / m \omega) e^{-i \omega t}\right] F_{n n^{\prime}}(\mathbf{p}, \mathbf{p}+\mathbf{k}) \\
& +\sum_{\mathbf{q}^{\prime}, n^{\prime \prime}}[V(q) n(\mathbf{q}, t)-U(q)] \\
& \times\left[F_{n^{\prime \prime} n^{\prime}}(\mathbf{p}, \mathbf{p}+\mathbf{k}-\mathbf{q}) N_{n^{\prime \prime} n}\right. \\
& \left.-F_{n n^{\prime \prime}}(\mathbf{p}+\mathbf{q}, \mathbf{p}+\mathbf{k}) N_{n n^{\prime}}\right] .
\end{aligned}
$$

Here $V\left(q_{y}, L\right)=4 \pi e^{2} I_{L}\left(q_{y} r\right) K_{L}\left(q_{y} r\right)$ is the Fourier transform of the e-e interaction, $I_{L}\left(K_{L}\right)$ is the modified Bessel function of the first (second) kind, and $U(q)$ is the Fourier component of $U\left(\mathbf{r}-\mathbf{R}_{i}\right)$. The electron density operator is written as $n(\mathbf{q})=\sum_{k, n n^{\prime}} F_{n n^{\prime}}(\mathbf{k}, \mathbf{k}+\mathbf{q}) N_{n n^{\prime}}\left(k_{y}, q_{y}, L\right)$, where $N_{n n^{\prime}}\left(k_{y}, q_{y}, L\right)$ is the matrix element between the two TB states, ${ }^{26}$ 
$N_{n n^{\prime}}\left(k_{y}, q, L\right)=\left\langle J+L, k_{y}+q, n\left|e^{i q_{y} y} e^{i L \phi}\right| J, k_{y}, n^{\prime}\right\rangle$.

The current density can be defined as $\mathbf{j}=\langle\delta H / \delta \mathbf{A}\rangle=\mathbf{j}_{0}$ $+\mathbf{j}_{1}$. Here $\mathbf{A}=(\mathbf{E} / i \omega) \exp (-i \omega t)$ is the vector potential and $\mathbf{j}_{0}=\left(\right.$ ine $\left.e^{2} / m \omega\right) \mathbf{E}=\sigma_{0}(\omega) \mathbf{E}$ is purely imaginary (where $n$ is the effective conduction electron concentration), $\mathbf{j}_{1}$ contains all the information on the e-e and e-i interactions; it can be written as

$$
\mathbf{j}_{1}(t)=\frac{e}{m} \sum_{\mathbf{p}} \sum_{n} \mathbf{p} F_{n n}(\mathbf{p}, \mathbf{p}, t) .
$$

Equation (1) is solved in two steps. ${ }^{27}$ We first calculate the static deviation of $F$ due to impurity scattering in the absence of the time-dependent field. The solution is then feed back to Eq. (1) to determine the linear response of $F$ to the external field. We obtain $\mathbf{j}_{1}(\omega)=(i e / m \omega) \Sigma_{\mathbf{q}} \mathbf{q} n(-\mathbf{q}, \omega) U(\mathbf{q})$. For random impurity with $r^{-1}$ potential, $U(q)=V(q) \sum_{i} e^{-i \mathbf{q} \cdot R_{i}}$, we obtain $\mathbf{j}(\omega)=\sigma(\omega) \mathbf{E}$, where $\sigma$ is the conductivity,

$$
\sigma(\omega)=\frac{\sigma_{0}(\omega)}{1+M(\omega) / \omega} .
$$

The memory function $M(\omega)$ is given as

$$
M(\omega)=\frac{1}{\omega} \frac{n_{I}}{n_{e}} \sum_{\mathbf{q}}|V(\mathbf{q})|^{2} q_{y}^{2}\left[\frac{1}{\varepsilon\left(q_{y}, L, 0\right)}-\frac{1}{\varepsilon\left(q_{y}, L, \omega\right)}\right] .
$$

Here $n_{I}\left(n_{e}\right)$ is the impurity (electron) concentration and $\varepsilon(q, \omega)$ is the dielectric function of the SWNT in the random phase approximation. ${ }^{22}$ By comparing Eq. (3) to the Drude type of conductivity, we identify the $f$-dependent photon absorption coefficient $\mathfrak{R} e[\sigma / \omega]=\sigma_{0} \Im m[M] / \omega^{2}$; the quantity $R(\omega)=\Im m[M(\omega)]$ is the usual transport scattering rate.

Both impurity scattering and photon absorption in SWNTs are strongly dependent on the size and chirality of the tube. If the tube is semiconducting, the main contribution at low to intermediate frequency is the single-particle interlevel excitations. If the tube is metallic, intraband plasmon excitation also contributes. In Fig. 1, we plot the real part $\left(\varepsilon_{R}\right)$ and the imaginary part $\left(\varepsilon_{I}\right)$ of the dielectric function and the dynamic structure factor of three zigzag SWNTs. For semiconducting $(10,0)$ and $(20,0)$ tubes, electrons are excited from the valence to the conduction bands of the same $J(L$ $=0)$. The $\varepsilon_{I}$ [Fig. 1(b)] is the absorption coefficient in the absence of impurity scattering. There exists a threshold excitation energy. For the metallic $(15,0)$ tube, the intraband transition channel is also open and the threshold energy is much lower. Both $\varepsilon_{R}$ and $\varepsilon_{I}$ can change drastically with the frequency, reflecting resonant and nonresonant couplings of electrons with photons.

In Fig. 2 we plot the scattering rate of the same three zigzag SWNTs, as a function of photon energy, for $n_{I} / n_{e}$ $=1 \%$. There is a striking difference between the rate of the metallic $(15,0)$ tube and semiconducting $(10,0)$ and $(20,0)$ tubes. For metallic tubes, the main contribution at low frequency is from the single-particle excitations. The scattering in the region of $\hbar \omega<0.5 \gamma_{0} \sim 1.5 \mathrm{eV}$ is very similar to that of a normal metal. The scattering due to the single-particle excitation is strongest at $\sim 0.6 \mathrm{eV}$, the characteristic energy of intraband plasmon excitation. The rapid decrease of the scattering after the first peak is mainly due to the fact that the intraband impurity scattering is inversely proportional to the frequency. Further increase of frequency will open up more channels through interband excitations. When the frequency

Downloaded 09 Jan 2007 to 130.130.37.12. Redistribution subject
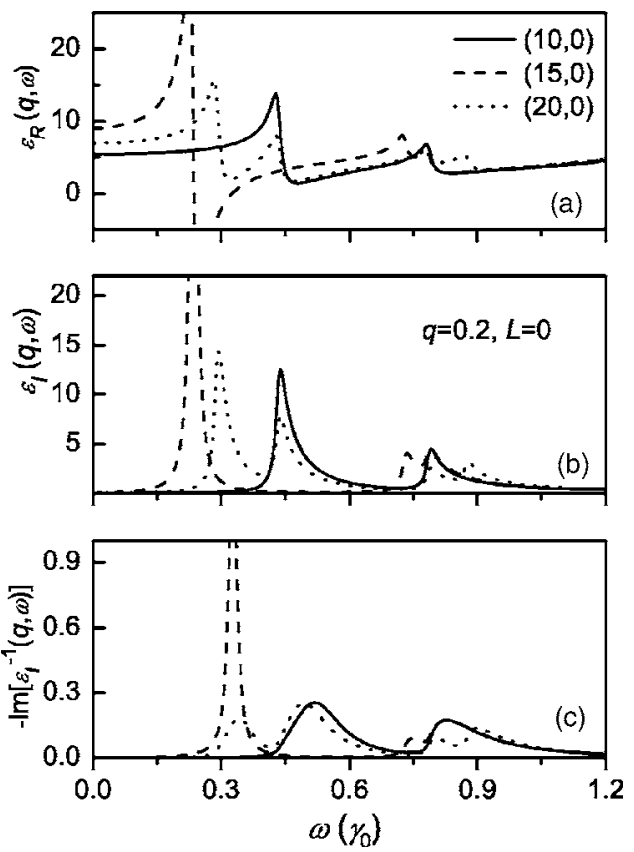

FIG. 1. Frequency dependent dielectric functions of three zigzag $(10,0)$, $(15,0)$, and (20) SWNTs. (a) Real part, (b) imaginary part, and (c) dynamic structural factor.

is close to the energy of the next interband transition, the scattering starts to increase again. For semiconducting tubes, the scattering due to the intraband excitation is absent at low frequencies. There is a threshold frequency, defined by the band gap, above which the scattering starts to increase. Figure 3 shows the rate in three metallic armchair SWNTs. The overall frequency dependence of the absorption is very similar to that in the metallic zigzag $(15,0)$ tube, as shown in Fig. 2.

Figures 2 and 3 demonstrate that the scattering exhibits strong size dependence. For the same type of SWNTs containing the same impurity density, a tube with a large diameter has less scattering, which is true for both semiconducting and metallic tubes. Our result is a clear demonstration of the size dependence of electron correlation and dynamic scattering in carbon nanotubes. The probability of forward

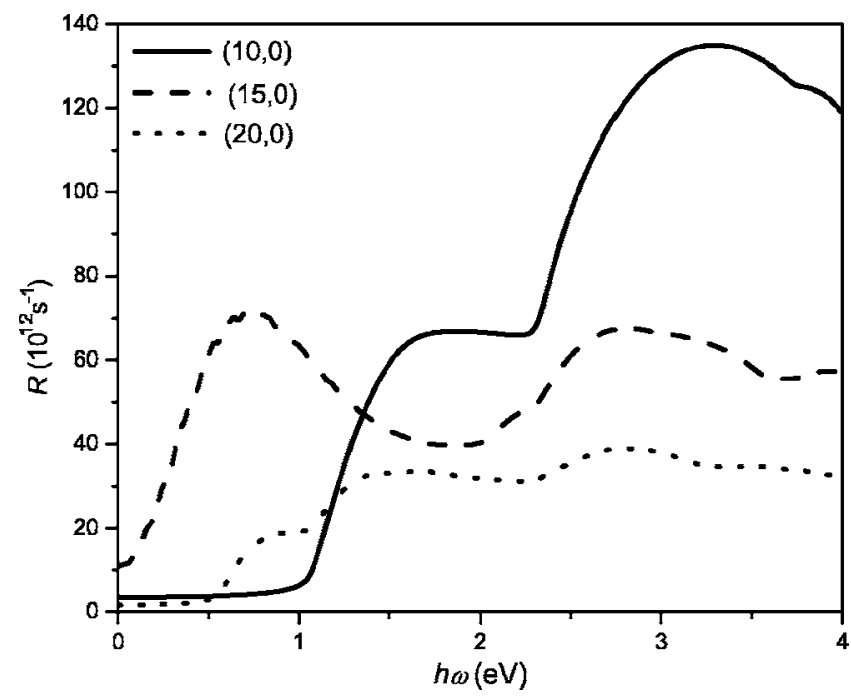

FIG. 2. Electron scattering rate of three zigzag $(10,0),(15,0)$, and $(20,0)$ SWNTs as a function of photon energy.

to AIP license or copyright, see http://apl.aip.org/apl/copyright.jsp 


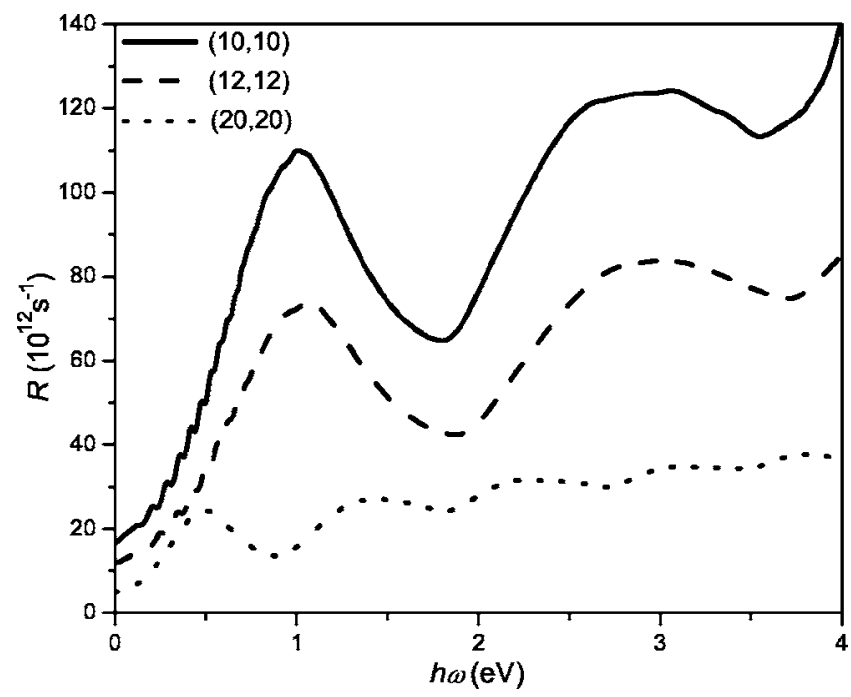

FIG. 3. Same as Fig. 2 for three armchair $(10,10),(12,12)$, and $(20,20)$ SWNTs.

scattering is smaller in small tubes than that in large tubes, because the smaller tubes behave closer to a one-dimensional system.

For perfect SWNTs, the absorption spectra contain discrete peaks separated by small tails [Fig. 1(b)], as shown by previous theories with or without many-body effect. ${ }^{10,18-21}$ The total absorption is the sum of the direct absorption and the impurity mediated absorption. The total photoresponse (PR) $\alpha(\omega)=\omega \epsilon_{I}(\omega)=\omega \epsilon_{I}^{0}(\omega)+4 \pi \sigma_{R}(\omega)$. Our results (Figs. 1-3) are based on microscopic models that do not contain any adjustable parameters. The total PR will be dependent on additional parameters such as the impurity concentration.

In Fig. 4, we plot the PR of a $(10,0)$ tube for two different impurity concentrations, $n_{I}=5 \%$ and $10 \%$ relative to $n_{e}$. The plasmon frequency of SWNT is taken to be $2.4 \gamma_{0}{ }^{26}$ The lifetime broadening is usually much smaller than the transport scattering rate. This is taken to be $0.05 \mathrm{eV}$ for $n_{I}=5 \%$ and $0.1 \mathrm{eV}$ for $n_{I}=10 \%$. The PR shows an absorption peak at the band gap and a large continuum beyond the gap. The

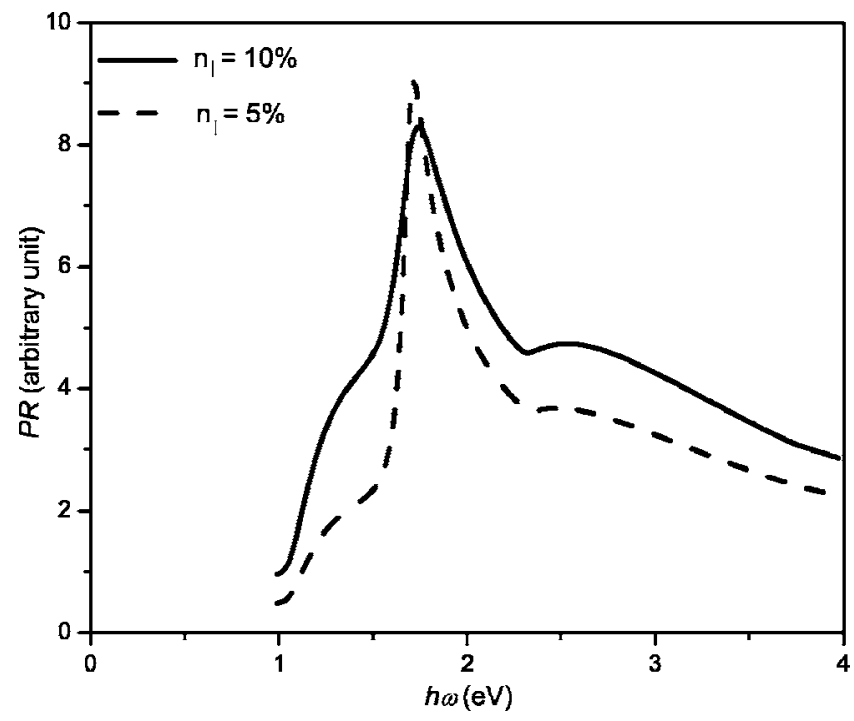

FIG. 4. Photoresponse of a $(10,0)$ tube for two different strengths of the random potentials. peak of the direct absorption decreases while the intensity of the continuum increases with increasing potential strength. To experimentally test our result, we suggest to perform optical absorption measurements in tubes with random defects. The other way to test this is to measure PR for tubes at helium temperature and at room temperature. We would expect the room temperature absorption to show a strong above-gap continuum due to random potentials (electronphonon interaction in this case).

In conclusion, we have formulated a quantum transport equation to account for the impurity-scattering-induced photon absorption in SWNTs. We demonstrate that even a very weak random impurity potential can add a significant continuum spectrum to the photon absorption. Our result indicates a universal nature of impurity mediated absorption.

The work is supported in part by the Australian Research Council, the US DOE, the National Fund for Distinguished Young Scholars of China (No. 60425415), NSF of China (No. 10390162), and the Shanghai Municipal Commission of Science and Technology.

${ }^{1}$ R. Saito, M. Fujita, G. Dresselhaus, and M. S. Dresselhaus, Appl. Phys. Lett. 60, 2204 (1992); Phys. Rev. B 46, 1804 (1992).

${ }^{2}$ J.-C. Charlier, Acc. Chem. Res. 35, 1063 (2002).

${ }^{3}$ D. P. Clougherty, Phys. Rev. Lett. 90, 035507 (2003).

${ }^{4}$ J.-Q. Lu, J. Wu, W. Duan, F. Liu, B. F. Zhu, and B. L. Gu, Phys. Rev. Lett. 90, 156601 (2003).

${ }^{5}$ J. Z. Cai, L. Lu, W. J. Kong, H. W. Zhu, C. Zhang, B. Q. Wei, D. H. Wu, and F. Liu, Phys. Rev. Lett. 97, 026402 (2006).

${ }^{6}$ S. J. Tans, A. R. M. Verschueren, and C. Dekker, Nature (London) 393, 49 (1998).

${ }^{7}$ R. Martel, T. Schmidt, H. R. Shea, T. Hertel, and P. Avouris, Appl. Phys. Lett. 73, 2447 (1998)

${ }^{8}$ J. Wu, J. Zhang, B. Larade, H. Guo, X. G. Gong, and F. Liu, Phys. Rev. B 69, 153406 (2004).

${ }^{9}$ M. Sergei, M. S. Strano, C. Kittrell, R. H. Hauge, R. E. Smalley, and R. B. Weisman, Science 298, 2361 (2002).

${ }^{10}$ Z. M. Li, Z. K. Tang, H. J. Liu, N. Wang, C. T. Chan, R. Saito, S. Okada, G. D. Li, J. S. Chen, N. Nagasawa, and S. Tsuda, Phys. Rev. Lett. 87, 127401 (2001)

${ }^{11}$ Y. Ohno, S. Kishimoto, T. Mizutani, T. Okazaki, and H. Shinohara, Appl. Phys. Lett. 84, 1368 (2004).

${ }^{12}$ A. Fujiwara, Y. Matsuoka, H. Suematsu, N. Ogawa, K. Miyano, H. Kataura, Y. Maniwa, S. Suzuki, and Y. Achiba, Jpn. J. Appl. Phys., Part 2 40, L1229 (2001).

${ }^{13}$ I. A. Levitsky and W. B. Euler, Appl. Phys. Lett. 83, 1857 (2003).

${ }^{14}$ K. Balasubramanian, Y. Fan, M. Burghard, K. Kern, M. Friedrich, U. Wannek, and A. Mews, Appl. Phys. Lett. 84, 2400 (2004).

${ }^{15}$ M. Freitag, Y. Martin, J. A. Misewich, R. Martel, and P. Avouris, Nano Lett. 3, 1067 (2003).

${ }^{16}$ A. Mohite, S. Chakraborty, P. Gopinath, G. U. Sumanasekera, and B. W. Alphenaar, Appl. Phys. Lett. 86, 061114 (2005).

${ }^{17}$ D.-H. Lien, W. K. Hsu, H. W. Zan, N. H. Tai, and C. H. Tsai, Adv. Mater. (Weinheim, Ger.) 18, 98 (2006).

${ }^{18}$ M. Machon, S. Reich, C. Thomsen, D. S. Portal, and P. Ordejon, Phys. Rev. B 66, 155410 (2002).

${ }^{19}$ H. J. Liu and C. T. Chan, Phys. Rev. B 66, 115416 (2002).

${ }^{20}$ G. Y. Guo, K. C. Chu, D. Wang, and C. Duan, Phys. Rev. B 69, 205416 (2004).

${ }^{21}$ C. D. Spataru, S. Ismail-Beigi, L. X. Benedict, and S. G. Louie, Phys. Rev. Lett. 92, 077402 (2004).

${ }^{22}$ M. F. Lin and D. S. Chuu, Phys. Rev. B 56, 4996 (1997).

${ }^{23}$ T. Stockli, J. M. Bonard, A. Chatelain, Z. L. Wang, and P. Stadelmann, Phys. Rev. B 64, 115424 (2001).

${ }^{24}$ G. Gumbs and G. R. Aizin, Phys. Rev. B 65, 195407 (2002).

${ }^{25}$ C. W. Chiu, C. P. Chang, F. L. Shyu, R. B. Chen, and M. F. Lin, Phys. Rev. B 67, 165421 (2003).

${ }^{26}$ K. W.-K. Shung, Phys. Rev. B 34, 979 (1986).

${ }^{27}$ C. Zhang, Phys. Rev. B 66, 081105(R) (2002). 